\title{
Novel Inter-Cell Interaction Approach for WCDMA-based Cognitive Networks
}

\author{
J. Nasreddine, O. Sallent, J. Pérez-Romero, R. Agustí \\ Dept. TSC, Universitat Politècnica de Catalunya (UPC) \\ c/ Jordi Girona, 1-3, Campus Nord, Barcelona, Spain \\ [jnassred, sallent, jorperez, ramon]@tsc.upc.edu
}

\begin{abstract}
In this paper, we propose a novel analytical framework that is able to capture inter-cell interactions and relevant variations in WCDMA systems using the so-called coupling matrix. Coupling matrix properties related to its spectral radius are analyzed and used to introduce a pertinent indicator which is able to dynamically optimize spectrum efficiency in the context of cognitive networks. Herein, an advanced spectrum management methodology based on coupling matrices is proposed as an illustrative example. Simulation results have shown that the proposed methodology has the capability to adapt to relevant changes in the network and outperforms the frequency allocation in classical hierarchical cell structure planning.
\end{abstract}

Keywords; ASM, cognitive network, coupling matrix, WCDMA

\section{INTRODUCTION}

The pervasive increase in multimedia services and the number of mobile users, as well as the proliferation of new radio access technologies, intensifies the need to revolutionize the existing vision of wireless communication, which is actually based on hermitic radio access networks (i.e. networks that do not interact). The new vision will be eventually based on the notion of cognitive heterogeneous networks where different radio accesses cooperate in order to dynamically find the best configuration satisfying both users and operators.

Cognitive networks exploit self-awareness and selfconfiguration capabilities to adapt to the unexpected changes in the radio environment. In the context of heterogeneous cognitive networks, WCDMA technique with its different duplex modes (e.g. TDD and FDD) and evolution (e.g. HSDPA and HSUPA) is still a concurrent technique. However, the current radio resource management and radio planning techniques will not be able to face the rising challenges and thus, new techniques should be developed. The realization of the new techniques in the context of cognitive networks is highly associated to the capability of the system to detect relevant changes that could alter its performance and to react to these changes by finding the suitable configuration. Therefore, metrics that can improve these capabilities are of high importance for the evolution of wireless networks. In this paper, we consider WCDMA network as a first step towards heterogeneous networks and we propose a new metric that offers important information for the cognitive network.
The main limiting problem in WCDMA systems, in terms of capacity and quality of service (QoS) levels, is the interference level, which depends on a plethora of correlated parameters with different distributions such as mobile positions, shadowing, cell loads, etc. These parameters lead to the concept of soft capacity and correlated QoS levels among users. Hence, a metric reflecting accurately these parameters could be of relevant interest for radio resource management (RRM) and advanced spectrum management (ASM) methodologies as well as cognitive networks.

Generally, the total received power in uplink and the total transmitted power in downlink as well as other QoS indicators such as outage and dropping probabilities are used to describe the status and performance of a system for a specific traffic distribution. Nevertheless, these indicators suffer considerable drawbacks. On the one hand, for low-loaded systems in which the majority of users are always satisfied, interference and power patterns could be considered as appropriate indicators. However, the outage probability is always null or very low in this case; that is no information can be derived from this indicator. On the other hand, in highly-loaded systems, the outage probability could be a relevant indicator to detect poor performance. However, it only reflects the macroscopic pattern of the system without giving any detail about inter-cell interaction that could help RRM and ASM methodologies to optimize system performance. Moreover, measured interference or power levels would give an optimistic perception of the real situation due to the fact that these levels will be constrained by the limitation on transmitted power. In order to overcome such drawbacks and limitations, we propose in this paper a smart indicator that can be used to capture the macroscopic and the microscopic patterns of all scenarios. The new indicator is denoted as coupling matrix and its objective is to reflect the inter-cell interaction using measurable parameters.

Recently, inter-cell interaction representation approaches were introduced to a limited extent in [4]-[7]. In [7], the authors have proposed an interesting quality indicator matrix assuming that the received interference of each cell can be isolated but without proposing an isolation scheme. Furthermore, uplink coupling matrices were proposed in [4][5] based on analytical approaches that use system measurements. In addition to the fact that our contribution proposes coupling matrices for both uplink and downlink, a 
relevant novelty included hereinafter is that the affecting properties of the coupling matrix are introduced and used for ASM methodologies in the context of cognitive networks. ASM methodologies aim at dynamically finding the best spectrum allocation over cells for all time periods, bearing in mind that traffic distribution suffers from periodic and unexpected variations over time and space [4][8].

In cognitive networks, as illustrated in Fig. 1, the cognition cycle could be represented by the following functionalities: observe, analyze, decide and act [1]-[3]. Moreover, the cognitive cycle includes a learning functionality. In the context of a WCDMA cognitive network, the embedded information in the coupling matrix could be the core of the analyzing functionality while the properties of this matrix can be the key information for the deciding functionality. Measurement data such as pathloss and $E_{b} / N_{0}$ are collected from the radio environment and used to build the coupling matrix which is averaged and aggregated over time and then saved in a database [4]. Periodically, the aggregated coupling matrix is analyzed and used together with analysis results to detect relevant variations and as entries to RRM and ASM algorithms. Moreover, this information, as well as key performance indicators (KPI), is sent to the learning functionality in order to ameliorate the performance of the optimization algorithm by tuning specific parameters.

The paper is organized as follows. In section II, coupling matrices are developed. Then, coupling matrix properties are analyzed with mathematical models in section III. Moreover, physical interpretations are introduced to explain these models. In section IV, we introduce an illustrative example where the coupling matrix is used by ASM methodologies. We conclude the paper with a summary and future works.

\section{COUPLING MATRIX}

Let us consider a WCDMA system with $K$ base stations and $F$ frequencies reflecting that operators use to have more than one frequency carrier in current WCDMA systems. The set of all base stations is called $\Lambda=\{j: j \in[1, K]\}$ and the set of cells operating with frequency carrier $f$ is called $\Lambda_{f}$. The number of users connected to base station $j$ is $n_{j}$. Furthermore, the set of all frequencies is called $\Phi=\{f: f \in[1, F]\}$. For given matrix $\boldsymbol{A}$ and vector $\boldsymbol{X}$, we refer throughout the paper by $(\boldsymbol{A} \boldsymbol{X})_{j}$ to the $j$-th element of vector $\boldsymbol{A X}$. Moreover, $(\boldsymbol{X})^{\mathbf{T}}$ represents the transpose of $\boldsymbol{X}$ and $\operatorname{diag}\left\{x_{1}, x_{2}, \ldots, x_{K}\right\}$ is a $K \times K$ diagonal matrix with diagonal elements $X_{i, i}=x_{i}$.

\section{A. Downlink}

In the downlink, the $E_{b} / N_{0}$ requirement for the $i$-th user of the $j$-th cell $i_{j}$ can be expressed as

$$
\left(\frac{E_{b}}{N_{o}}\right)_{i_{j}}=\frac{\frac{P_{T, i_{j}}}{L_{i_{j}, j}} \times \Theta_{i_{j}}}{N_{0, D}+\chi_{i_{j}}+\alpha_{D} \times\left[\frac{P_{T, j}-P_{T, i_{j}}}{L_{i_{j}, j}}\right]}
$$

where $P_{T, i_{j}}$ is the power devoted to $i_{j}, \chi_{i_{j}}$ represents the inter- cell interference experienced by mobile $i_{j}, \Theta_{i_{j}}$ is the spreading factor of mobile $i_{j}$ (i.e. the ratio between chip rate and bit rate), $L_{i_{j}, j}$ is the pathloss between mobile $i_{j}$ and base station $j$,

$\alpha_{D}$ is the orthogonality factor in downlink $\left(\alpha_{D}=0\right.$ for perfect orthogonality and 1 for non-orthogonality), $N_{0, D}$ is the downlink background noise power, and $P_{T, j}$ is the total transmitted power by base station $j$ and it is given by

$$
P_{T, j}=P_{p, j}+\sum_{i_{j}=1}^{n_{j}} P_{T, i_{j}}
$$

where $P_{p, j}$ is the power devoted to common control channels in cell $j$.

From (1) and (2), we get

$$
P_{T, j}=\frac{P_{p, j}+\sum_{i_{j}=1}^{n_{j}} \frac{\left(N_{0, D}+\chi_{i_{j}}\right)}{\varepsilon_{i_{j}}+\alpha_{D}} L_{i_{j}, j}}{1-\sum_{i_{j}=1}^{n_{j}} \frac{\alpha_{D}}{\varepsilon_{i_{j}}+\alpha_{D}}}
$$

where $\varepsilon_{i_{j}}$ is defined by the ratio $\Theta_{i_{j}} /\left(E_{b} / N_{0}\right)_{i_{j}}$. Moreover, inter-cell interference $\chi_{i_{j}}$ could be written as:

$$
\chi_{i_{j}}=\sum_{l \in \Lambda-\{j\}} \frac{P_{T, l}}{L_{i_{j}, l}} .
$$

Thus, $\chi_{i_{j}}$ includes all interference power received by mobile $i_{j}$ from all base stations except base station $j$. By substituting (4) in (3), we get

$$
P_{T, j}=\frac{P_{p, j}+N_{0, D} D_{j}+\sum_{l \in \Lambda-\{j\}} P_{T, l} S_{j, l}}{1-\alpha_{D} S_{j, j}}
$$

where

$$
\begin{aligned}
S_{j, l} & =\sum_{i_{j}=1}^{n_{j}} \frac{L_{i_{j}, j}}{L_{i_{j}, l}} \frac{1}{\varepsilon_{i_{j}}+\alpha_{L}} \\
D_{j} & =\sum_{i_{j}=1}^{n_{j}} \frac{L_{i_{j}, j}}{\varepsilon_{i_{j}}+\alpha_{L}}
\end{aligned}
$$

In these definitions, $\alpha_{L}$ is the orthogonality factor in link direction $L$, which could be either uplink $(U)$ or downlink $(D)$.

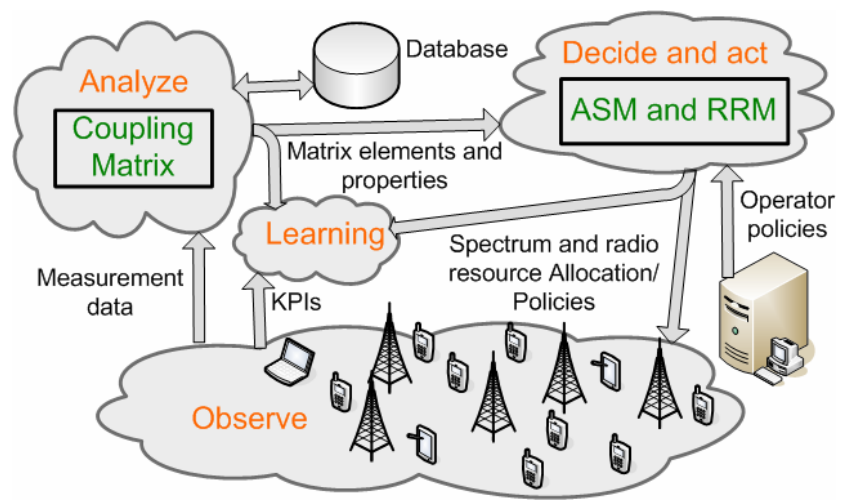

Figure 1. Integration of coupling matrix in cognitive network 
The coupling existing in a WCDMA cellular system is explicitly reflected in (5), where the resulting transmitted power level by a given base station depends on all other base stations' respective transmitted power levels.

The vector of total transmitted powers $\boldsymbol{P}_{\boldsymbol{T}}=\left(P_{T, 1}, P_{T, 2}, \ldots, P_{T, K}\right)^{\mathrm{T}}$ satisfying the system of equations (5) can be interpreted as the fixed point of mapping $\mathcal{F}_{D}$ :

$$
\begin{aligned}
\mathcal{F}_{D}: & \mathbb{R}_{+}^{K} \rightarrow \mathbb{R}_{+}^{K} \\
\boldsymbol{P}_{\boldsymbol{T}} & \rightarrow \boldsymbol{J}=\boldsymbol{C}^{\boldsymbol{D}} \boldsymbol{P}_{\boldsymbol{T}}+\boldsymbol{P}_{N}^{\boldsymbol{D}}
\end{aligned}
$$

where $\boldsymbol{P}_{N}^{D}$ is defined by its elements

$$
P_{N, j}^{D}=\left(P_{p, j}+N_{0, D} D_{j}\right) /\left(1-\alpha_{D} S_{j, j}\right) .
$$

In (7), we highlight that the Jacobian $\boldsymbol{C}^{\boldsymbol{D}}$ of mapping $\mathcal{F}_{D}$ is by definition the matrix of all partial derivatives of vector $\boldsymbol{J}$ in function of the elements of vector $\boldsymbol{P}_{T}$ and it is defined by

$$
C_{j, l}^{D}=\frac{\partial J_{j}}{\partial P_{T, l}}=\left\{\begin{array}{cc}
0 & \text { if } l=j \\
\frac{S_{j, l}}{1-\alpha_{D} S_{j, j}} & \text { otherwise }
\end{array}\right.
$$

Each element of coupling matrix $\boldsymbol{C}^{\boldsymbol{D}}$ represents the impact of the total transmitted power by one base station on the total transmitted power by another base station.

\section{B. Uplink}

In the following, $I_{j}$ stands for the total power received by base station $j$ and it is given by:

$$
I_{j}=\chi_{j}+P_{R, j}+N_{0, U}
$$

where $\chi_{j}$ and $P_{R, j}$ are the inter-cell interference and the total own-cell received power by cell $j$ and $N_{0, U}$ is uplink background noise power.

In uplink, the $E_{b} / N_{0}$ requirement for user $i_{j}$ can be written as

$$
\left(\frac{E_{b}}{N_{0}}\right)_{i_{j}}=\frac{P_{j, i_{j}} \Theta_{i_{j}}}{\left[P_{R, j}-P_{j, i_{j}}\right]+\chi_{j}+N_{0, U}}
$$

where $P_{j, i_{j}}$ is the useful received power by base station $j$ from mobile $i_{j}$. We emphasize here that the orthogonality factor is equal to unity $\left(\alpha_{U}=1\right)$. It was shown in [5] that by using (11) and having $\alpha_{U}=1$ in $S_{l, j}$ and $S_{j, j}$, we can write

$$
I_{j}=\frac{N_{0, U}+\sum_{l \in \Lambda-\{j\}} S_{l, j} I_{l}}{1-S_{j, j}} \quad \forall j \in \Lambda .
$$

The vector of total received powers $\boldsymbol{I}=\left(I_{1}, I_{2}, \ldots, I_{K}\right)^{\mathrm{T}}$ satisfying the system of equations (12) can be interpreted as the fixed point of mapping $\mathcal{F}_{U}$ :

$$
\begin{aligned}
\mathcal{F}_{U}: & \mathbb{R}_{+}^{K} \\
\boldsymbol{I} & \rightarrow \mathbb{R}_{+}^{K} \\
& \rightarrow \boldsymbol{J}=\boldsymbol{C}^{\boldsymbol{U}} \boldsymbol{I}+\boldsymbol{P}_{N}^{U}
\end{aligned}
$$

where $\boldsymbol{P}_{N}^{U}$ is defined by. its elements

$$
P_{N, j}^{U}=N_{0, U} /\left(1-S_{j, j}\right) .
$$

In (13), we highlight that the Jacobian $\boldsymbol{C}^{\boldsymbol{U}}$ of mapping $\mathcal{F}_{U}$ is by definition the matrix of all partial derivatives of vector $\boldsymbol{J}$ in function of the elements of vector $\boldsymbol{I}$ and it is defined by

$$
C_{j, l}^{U}=\frac{\partial J_{j}}{\partial I_{l}}=\left\{\begin{array}{cc}
0 & \text { if } l=j \\
\frac{S_{l, j}}{1-S_{j, j}} & \text { otherwise }
\end{array} .\right.
$$

Each element of coupling matrix $\boldsymbol{C}^{\boldsymbol{U}}$ represents the impact of the total received power by one base station on the total received power by another base station.

\section{Generic Form}

The coupling matrix in both links can be written as

$$
\begin{aligned}
& \boldsymbol{C}^{\boldsymbol{D}}=\operatorname{diag}\left\{1 /\left(1-\alpha_{D} S_{1,1}\right), 1 /\left(1-\alpha_{D} S_{2,2}\right), \cdots, 1 /\left(1-\alpha_{D} S_{K, K}\right)\right\} \boldsymbol{S}^{\boldsymbol{D}} \\
& \boldsymbol{C}^{\boldsymbol{U}}=\operatorname{diag}\left\{1 /\left(1-S_{1,1}\right), 1 /\left(1-S_{2,2}\right), \cdots, 1 /\left(1-S_{K, K}\right)\right\}\left(\boldsymbol{S}^{\boldsymbol{U}}\right)^{\mathrm{T}}
\end{aligned}
$$

where $S^{L}$ is a $K \times K$ square matrix whose $(j, l)$ element is $S_{j, l}$. We emphasize here, that the elements of $\boldsymbol{S}^{\boldsymbol{U}}$ and $\boldsymbol{S}^{\boldsymbol{D}}$ differ by the value of $\alpha_{L}$ which is always unity in uplink. Herein, matrix $S^{L}$ represents the pure interaction between the different cells while the diagonal matrix reflects the internal loads of the different cells. Each row of matrix $\boldsymbol{S}^{L}$ represents the impact of one cell on the other cells while each column represents the experienced impact by one cell from the other cells. Moreover, (5) and (12) could be written in the following matrix form:

$$
\boldsymbol{V}=\boldsymbol{C}^{L} \boldsymbol{V}+\boldsymbol{P}_{N}^{L}
$$

where $\boldsymbol{V}$ is a $K \times 1$ vector that replaces $\boldsymbol{I}$ in uplink and $\boldsymbol{P}_{\boldsymbol{T}}$ in downlink and $L$ is the link direction. For the sake of simplicity, we use $\boldsymbol{C}, \alpha$ and $\boldsymbol{P}_{N}$ instead of $\boldsymbol{C}^{\boldsymbol{L}}, \alpha_{L}$ and $\boldsymbol{P}_{N}^{\boldsymbol{L}}$ when no confusion appears and (17) becomes

$$
\boldsymbol{V}=\boldsymbol{C} \boldsymbol{V}+\boldsymbol{P}_{N} \text {. }
$$

\section{COUPling MATrix PROPERTIES}

By using the same methodology of [9], it is easy to show that coupling matrix $\boldsymbol{C}$ is a non-negative irreducible matrix. Hence, matrix $\boldsymbol{C}$ has interesting properties inspired from (18) that can be used as performance indicators.

In order to study the properties of coupling matrix $\boldsymbol{C}$, we first introduce some useful definitions.

Definition 1. Let $\boldsymbol{A}=\left[a_{i, j}\right]$ and $\boldsymbol{B}=\left[b_{i, j}\right]$ be $m \times n$ matrices, then

$$
\mathrm{A} \geq \mathrm{B} \leftrightarrow a_{i, j} \geq b_{i, j} \forall i, j .
$$

Definition 2. A non-constrained WCDMA system (i.e. a system without power limitations) is called feasible if all users could be served with the required $E_{b} / N_{0}$ using finite positive powers. That is, there exists a positive vector $\boldsymbol{V}$ that satisfies

$$
\boldsymbol{V} \geq \boldsymbol{C} \boldsymbol{V}+\boldsymbol{P}_{\boldsymbol{N}} .
$$

Equation (19) can be deduced from (1), (11) and (18) by assuming that users' QoS levels are higher or equal to the required value (i.e. equalities (1) and (11) become inequalities). However, the power vector of (18) is the most suitable solution in terms of power consumption since it is the minimum vector that achieves the desired QoS levels (Corollary 1 in [10]).

Definition 3. The spectral radius $\rho(\boldsymbol{C})$ of a $K \times K$ matrix $\boldsymbol{C}$ is the eigenvalue with the maximum modulus; that is, 


$$
\rho(\boldsymbol{C})=\max _{j \in \Lambda}\left|\lambda_{j}\right|
$$

where $\lambda_{1}, \lambda_{2}, \ldots, \lambda_{K}$ are the eigenvalues of matrix $\boldsymbol{C}$.

Spectral radius $\rho(\boldsymbol{C})$ of the coupling matrix has paramount impact on the interference and transmitted power patterns and thus on system performance (e.g. outage probability, dropping probability, etc.). The analysis of matrix properties is inspired from the proposals in [11] and [12].

Theorem 1. A non-constrained system characterized by the following relation

$$
\boldsymbol{V}=\boldsymbol{C} \boldsymbol{V}+\boldsymbol{P}_{\boldsymbol{N}}
$$

is feasible if and only if the spectral radius of matrix $\boldsymbol{C}$ is less than unity $(\rho(\boldsymbol{C})<1)$.

Proof First, we prove that if $\rho(\boldsymbol{C})<1$, then the system is feasible (i.e. there is a positive power vector satisfies (21)). Since $\boldsymbol{P}_{\boldsymbol{N}}>0$, we should only prove that $\left(\mathfrak{I}_{\boldsymbol{K}}-\boldsymbol{C}\right)$ is invertible and its inverse is positive.

For a non-negative irreducible square matrix $\boldsymbol{C}$ and any positive constant $c$, we have the following equivalent (theorem 3.9 in [15]):

If $c>\rho(\boldsymbol{C}) \leftrightarrow\left(c \mathfrak{I}_{K^{-}} \boldsymbol{C}\right)$ is non-singular and $\left(c \mathfrak{I}_{\boldsymbol{K}^{-}} \boldsymbol{C}\right)^{-1}>0$ where $\mathfrak{I}_{K}$ is $(K \times K)$ identity matrix. Setting $c$ to one and having $\rho(\boldsymbol{C})<1$, we get $\left(\mathfrak{I}_{\boldsymbol{K}^{-}} \boldsymbol{C}\right)$ is non-singular and $\left(\mathfrak{I}_{\boldsymbol{K}^{-} \boldsymbol{C}}\right)^{-1}>0$.

Second, we prove that if $\rho(\boldsymbol{C})>1$, then (21) is not feasible and at least one element of vector $\boldsymbol{V}$, which satisfies either (19) or (21), is negative. Indeed, the Perron-Frobenius theorem states that the minimum real $\lambda$, that satisfies $\lambda \boldsymbol{V} \geq \boldsymbol{C} \boldsymbol{V}$ with a positive vector $\boldsymbol{V}$ is the spectral radius $\rho(\boldsymbol{C})$ [13]. Since, $\rho(\boldsymbol{C})>1$, then

$$
\exists j \in \Lambda, \quad V_{j}<(\boldsymbol{C} \boldsymbol{V})_{j} .
$$

Therefore, there is no positive power vector $\boldsymbol{V}$ that satisfies $\boldsymbol{V} \geq \boldsymbol{C} \boldsymbol{V}$ and thus there is no positive power vector that satisfies $\boldsymbol{V} \geq \boldsymbol{C} \boldsymbol{V}+\boldsymbol{P}_{\boldsymbol{N}}$ for a positive $\boldsymbol{P}_{N}$

Lemma 1. Let $\boldsymbol{C}$ be a $K x K$ non-negative irreducible matrix and $\rho(\boldsymbol{C})$ its spectral radius. Then, for any $K \times 1$ positive vector $\boldsymbol{V}$

$$
\exists j \in \Lambda, \quad(\boldsymbol{C} \boldsymbol{V})_{j} \geq \rho(\boldsymbol{C}) V_{j} .
$$

Proof Assume that $\boldsymbol{C} \boldsymbol{V}<\rho(\boldsymbol{C}) \boldsymbol{V}$. Since $\boldsymbol{C}$ is irreducible nonnegative matrix, there exist a $1 \times K$ positive left eigenvector $\boldsymbol{E}^{\mathbf{T}}$. Multiplying both sides of the above inequality by $\boldsymbol{E}^{\mathrm{T}}$, we get

$$
\boldsymbol{E}^{\mathrm{T}} \boldsymbol{C} \boldsymbol{V}<\boldsymbol{E}^{\mathrm{T}} \rho(\boldsymbol{C}) \boldsymbol{V} .
$$

Since $\boldsymbol{E}^{\mathbf{T}}$ is a left eigenvector of $\boldsymbol{C}$, then $\boldsymbol{E}^{\mathbf{T}} \boldsymbol{C}=\boldsymbol{E}^{\mathbf{T}} \rho(\boldsymbol{C})$ and

$$
\boldsymbol{E}^{\mathbf{T}} \rho(\boldsymbol{C}) \boldsymbol{V}<\boldsymbol{E}^{\mathbf{T}} \rho(\boldsymbol{C}) \boldsymbol{V}
$$

which is impossible. Hence, the assumption is not true and there exists index $j$ for which $(\boldsymbol{C V})_{j} \geq \rho(\boldsymbol{C}) V_{j}$

In the following, we consider that the spectral radius is always less than unity. This is coherent with operational systems since the opposite scenario leads to unacceptable QoS levels. Indeed, when the spectral radius is higher than unity, the system cannot satisfy all users even without power constraints. Moreover, radio resource management procedures yield indirectly a decrease in the spectral radius since these procedures aim at reducing interference profile.

\section{A. Spectral Radius Relationships}

When representing a system by a non-negative irreducible matrix, spectral radius could be seen as relevant indicator to capture the overall behavior of the system. Indeed, a pervasive increase in matrix elements (or an increase of any element) leads necessarily to an increase in the spectral radius [13] of a non-negative irreducible matrix. In addition to this direct property, the following theorems will emphasize the importance of the spectral radius in detecting important changes in the system as well as reflecting its actual state.

Theorem 2. Suppose that the non-constrained system characterized by (21) is feasible by using vector $\boldsymbol{V}^{*}$. Then,

$$
\exists j \in \Lambda, \quad V_{j}^{*} \geq \frac{P_{N, j}}{1-\rho(\boldsymbol{C})} .
$$

Proof For any positive vector $\boldsymbol{V}$ there exists an index $j$ for which $(\boldsymbol{C} \boldsymbol{V})_{j} \geq \rho(\boldsymbol{C}) V_{j}$ (lemma 1). Hence, we apply this result to (21) using the positive vector $\boldsymbol{V}^{*}$ and we get

$$
\exists j \in \Lambda, \quad V_{j}^{*} \geq \rho(\boldsymbol{C}) V_{j}^{*}+P_{N, j} .
$$

By using simple mathematical manipulation and the fact that $\rho(\boldsymbol{C})<1$ since the system is feasible (theorem 1), we get

$$
\exists j \in \Lambda, \quad V_{j}^{*} \geq \frac{P_{N, j}}{1-\rho(\boldsymbol{C})} .
$$

Corollary 1. Suppose that the non-constrained system characterized by (21) is feasible by using vector $\boldsymbol{V}^{*}$. Then

$$
\exists j \in \Lambda, \quad \max _{l \in \Lambda}\left(V_{l}^{*}\right) \geq \frac{P_{N, j}}{1-\rho(\boldsymbol{C})} .
$$

Proof From theorem 2, we have

$$
\exists j \in \Lambda, \quad V_{j}^{*} \geq \frac{P_{N, j}}{1-\rho(\boldsymbol{C})} .
$$

Then,

$$
\max _{l \in \Lambda}\left(V_{l}^{*}\right) \geq V_{j}^{*} \geq \frac{P_{N, j}}{1-\rho(\boldsymbol{C})} .
$$

Interpretation The above theorem and corollary show that the value of the total transmitted power in downlink and the total received power in uplink corresponding to the worst cell (i.e. $\left.\arg \max _{l \in \Lambda}\left(V_{l}^{*}\right)\right)$ if no power constraints are applied, is lower-bounded by a parameter that depends on the spectral radius and internal load $S_{j, j}$ that appears in the denominator of $P_{N, j}$ as we can see in (8) and (14). The interesting issue in this property is that the system would be able to easily estimate the unconstrained total transmitted powers by base stations in downlink and the unconstrained total received power in uplink. Note that these power levels cannot be measured directly by the system due to power limitation constraints. Furthermore, these power levels reflect the actual state of the system more accurately than the actual measured powers, which are truncated by the power limitation. Hence, the estimated levels could be of high interest to evaluate the system behavior. Inspired by the results of corollary 1, we performed system level simulations and we found that the maximum unconstrained transmitted power would be in most 
cases higher than the lower bound $\eta=\max _{j \in \Lambda}\left[P_{N, j} /(1-\rho(\boldsymbol{C}))\right]$ which can be seen in Fig. 2, where we show the maximum unconstrained total transmitted power $P$ among all base stations of the layout defined later by Fig. 3 as a function of $\eta$. The results are obtained using Montecarlo simulation where mobile densities are 40 mobiles $/ \mathrm{km}^{2}$ and 880 mobiles $/ \mathrm{km}^{2}$ in macro- and micro-cells respectively. The obtained correlation between the two metrics is 0.9 . These results show that $\eta$ is a good estimation of $P$ and could be used to detect situations where the system cannot satisfy all users using the available power. Indeed, the maximum transmitted power is at maximum $2 \mathrm{~dB}$ higher than $\eta$ as depicted in Fig. 2. Therefore, it will be used in the ASM illustrative example of section IV.

\section{B. Impact of Matrix Elements' Variations}

In this section, we will use coupling matrix properties to estimate power vector changes when the system experiences fluctuations in mobile pathlosses. These fluctuations are expressed by changes in the values of parameters $S_{j, k}$ and $D_{j}$. In the following, we assume that variations $\Delta S_{j, k}$ of parameters $S_{j, k}$ and $\Delta D_{j}$ of parameters $D_{j}$ are independent. This could be justified by the fact that pathlosses to different base stations could be considered as independent.

Lemma 2. Suppose that the non-constrained system characterized by (21) is feasible by using vector $\boldsymbol{V}^{*}$. For independant increases $\Delta S_{j, k}$ and $\Delta D_{j}$, the derivative of each element in the power vector in downlink is ascertained as

$$
\left.\frac{\partial V_{i}}{\partial S_{j, k}}\right|_{V=V^{*}}= \begin{cases}\left.\sum_{l \in \Lambda} C_{i, l} \frac{\partial V_{l}}{\partial S_{j, k}}\right|_{V=V^{*}}+\frac{V_{k}}{1-\alpha S_{i, i}} & \text { if } j=i \text { and } k \neq i \\ \left.\sum_{l \in \Lambda} C_{i, l} \frac{\partial V_{l}}{\partial S_{j, k}}\right|_{V=V^{*}} & \text { if } j \neq i \\ \left.\sum_{l \in \Lambda} C_{i, l} \frac{\partial V_{l}}{\partial S_{j, k}}\right|_{V=V^{*}}+\frac{\alpha V_{i}}{1-\alpha S_{i, i}} & \text { if } j=k=i\end{cases}
$$

and

$$
\frac{\partial V_{i}}{\partial D_{j}}= \begin{cases}\left.\sum_{l \in \Lambda} C_{i, l} \frac{\partial V_{l}}{\partial D_{j}}\right|_{V=V^{*}}+\frac{N_{0, D}}{1-\alpha S_{i, i}} & \text { if } j=i \\ \left.\sum_{l \in \Lambda} C_{i, l} \frac{\partial V_{l}}{\partial D_{j}}\right|_{V=V^{*}} & \text { Otherwise }\end{cases}
$$

In uplink, $\alpha$ is set to $1, D_{i}$ to 1 and $P_{p . i}$ to 0 . Moreover, $j$ and $k$ are swapped in the right-hand side of (25).

Proof Since we have assumed that the increases in different $S_{j, k}$ are independent, then

and

$$
\frac{\partial S_{l, i}}{\partial S_{j, k}}= \begin{cases}1 & \text { if } l=j \text { and } i=k \\ 0 & \text { Otherwise }\end{cases}
$$

$$
\frac{\partial S_{l, i}}{\partial D_{j}}=\frac{\partial D_{j}}{\partial S_{l, i}}=0 \quad \forall i, j, l \in \Lambda .
$$

Using (9) and (18) as well as the definition of $\boldsymbol{P}_{N}$, all the derivatives can be directly extracted.

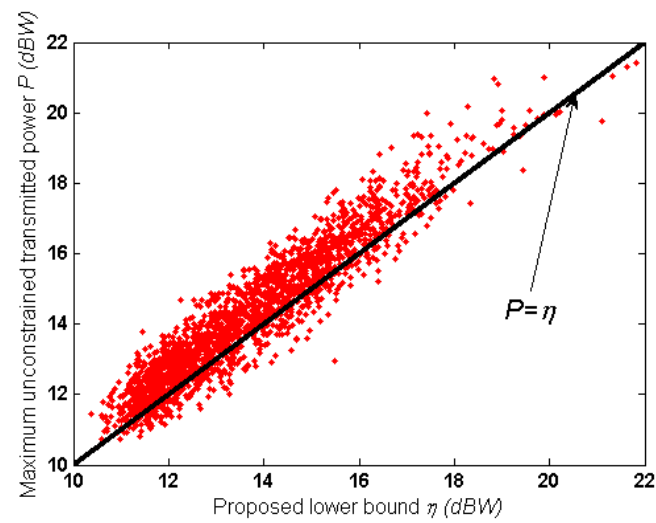

Figure 2.Correlation between the maximum unconstrained transmitted power and $\eta$

Lemma 3. In a non-constrained feasible system where parameters $S_{j, k}$ and $D_{j}$ are time variables and their variations are independent, the derivatives of the power vector in function of these parameters are interrelated by

$$
\begin{array}{ll}
\sum_{j \in \Lambda}\left(\left.\sum_{k \in \Lambda} \Delta S_{j, k} \frac{\partial \boldsymbol{V}}{\partial S_{j, k}}\right|_{V=V^{*}}+\left.\Delta D_{j} \frac{\partial \boldsymbol{V}}{\partial D_{j}}\right|_{V=V^{*}}\right)=\left(\mathfrak{I}_{K}-\boldsymbol{C}\right)^{-1} \boldsymbol{\Psi}_{\boldsymbol{D}} & \text { In downlink } \\
\left.\sum_{j \in \Lambda} \sum_{k \in \Lambda} \Delta S_{j, k} \frac{\partial \boldsymbol{V}}{\partial S_{j, k}}\right|_{V=\boldsymbol{V}^{*}}=\left(\mathfrak{I}_{K}-\boldsymbol{C}\right)^{-1} \boldsymbol{\Psi}_{\boldsymbol{U}} & \text { In uplink }
\end{array}
$$

where $\Psi_{D}$ is a $K x 1$ vector defined by

$$
\Psi_{D, i}=\frac{\alpha \Delta S_{i, i} V_{i}+\sum_{l \in \Lambda-\{i\}} \Delta S_{i, l} V_{l}+\Delta D_{i} N_{0, D}}{1-\alpha S_{i, i}}
$$

and $\Psi_{U}$ is a $K x 1$ vector defined by

$$
\Psi_{U, i}=\frac{\Delta S_{i, i} V_{i}+\sum_{l \in \Lambda-\{i\}} \Delta S_{l, i} V_{l}}{1-S_{i, i}} .
$$

Proof Using lemma 2 for downlink, we can directly write

$$
\begin{aligned}
& \left.\frac{\partial \boldsymbol{V}}{\partial S_{j, k}}\right|_{\boldsymbol{V}=\boldsymbol{V}^{*}}=\left.\boldsymbol{C} \frac{\partial \boldsymbol{V}}{\partial S_{j, k}}\right|_{\boldsymbol{V}=\boldsymbol{V}^{*}}+\left(1-\beta_{j, k}\right) \mathbf{O}_{1}^{(j, k)} \\
& \left.\frac{\partial \boldsymbol{V}}{\partial D_{j}}\right|_{\boldsymbol{V}=\boldsymbol{V}^{*}}=\left.\boldsymbol{C} \frac{\partial \boldsymbol{V}}{\partial D_{j}}\right|_{\boldsymbol{V}=\boldsymbol{V}^{*}}+\mathbf{O}_{2}^{(j)}
\end{aligned}
$$

where $\beta_{j, k}$ is the $(j, k)$ element of the $K \times K$ diagonal matrix $\operatorname{diag}\{1-\alpha, 1-\alpha, \ldots, 1-\alpha\}$, and $\mathbf{O}_{1}^{(j, k)}$ and $\mathbf{O}_{2}^{(j)}$ are $K \times 1$ vectors whose elements are equal to 0 except the $j$-th element which is defined by $\mathrm{O}_{1, j}^{(j, k)}=V_{k} /\left(1-\alpha S_{j, j}\right)$ and $\mathrm{O}_{2}^{(j)}=N_{0, D} /\left(1-\alpha S_{j, j}\right)$.

Using simple mathematical manipulations on (30), we get

$$
\begin{gathered}
\left.\left(\mathfrak{I}_{\boldsymbol{K}}-\boldsymbol{C}\right) \frac{\partial \boldsymbol{V}}{\partial S_{j, k}}\right|_{\boldsymbol{V}=\boldsymbol{V}^{*}}=\left(1-\beta_{j, k}\right) \mathbf{O}_{1}^{(j, k)} \\
\left.\left(\mathfrak{I}_{\boldsymbol{K}}-\boldsymbol{C}\right) \frac{\partial \boldsymbol{V}}{\partial D_{j}}\right|_{\boldsymbol{V}=\boldsymbol{V}^{*}}=\mathbf{O}_{2}^{(j)}
\end{gathered}
$$

For a fixed $j$ and for all $k$, we multiply each of equations (31) by $\Delta S_{j, k}$ and we sum over all $k$. Then we sum it to the multiplication of (32) by $\Delta D_{j}$ and we get 


$$
\left.\left(\mathfrak{I}_{K}-\boldsymbol{C}\right) \sum_{k \in \Lambda} \Delta S_{j, k} \frac{\partial \boldsymbol{V}}{\partial S_{j, k}}\right|_{V=V^{*}}+\left.\Delta D_{j} \frac{\partial \boldsymbol{V}}{\partial D_{j}}\right|_{V=V^{*}}=\mathbf{O}^{(j)}
$$

where $\mathbf{O}^{(j)}$ is the sum of $\mathbf{O}_{1}^{(j, k)}$ over all $k$ and $\mathbf{O}_{2}^{(j)}$; thus the elements of $\mathbf{O}^{(j)}$ are equal to 0 except the $j$-th element which is defined by

$$
\mathrm{O}_{j}^{(j)}=\frac{\sum_{k \in \Lambda}\left[\left(1-\beta_{j, k}\right) \Delta S_{j, k} V_{k}\right]+\Delta D_{j} N_{0, D}}{1-\alpha S_{j, j}} .
$$

Now, summing over $j$, we obtain

$$
\left(\mathfrak{I}_{K}-\boldsymbol{C}\right) \sum_{j \in \Lambda}\left(\left.\sum_{k \in \Lambda} \Delta S_{j, k} \frac{\partial \boldsymbol{V}}{\partial S_{j, k}}\right|_{V=V^{*}}+\left.\Delta D_{j} \frac{\partial \boldsymbol{V}}{\partial D_{j}}\right|_{V=V^{*}}\right)=\Psi_{\boldsymbol{D}} .
$$

Since the system is feasible, then $\mathfrak{I}_{K^{-}} \boldsymbol{C}$ is invertible (theorem 1) and thus we obtain equation (27) for downlink. The same steps can be used to obtain the uplink equation

Corollary 2. Suppose that the non-constrained system characterized by (21) is feasible by using vector $\boldsymbol{V}^{*}$. For small increases $\Delta S_{j, k}$ of parameters $S_{j, k}$, and $\Delta D_{j}$ of parameters $D_{j}$, we get a variation $\Delta \boldsymbol{V}$ in power vectors that satisfies:

$$
\left(\mathfrak{I}_{K}-C^{L}\right) \Delta V \approx \Psi_{L}
$$

Proof Taylor series approximation of first order for vector $\boldsymbol{V}$ with respect to $S_{j, k}$ and $D_{j}$ gives

$$
\Delta \boldsymbol{V} \approx \begin{cases}\sum_{j \in \Lambda}\left(\left.\sum_{k \in \Lambda} \Delta S_{j, k} \frac{\partial \boldsymbol{V}}{\partial S_{j, k}}\right|_{V=V^{*}}+\left.\Delta D_{j} \frac{\partial \boldsymbol{V}}{\partial D_{j}}\right|_{V=V^{*}}\right) & \text { In downlink } \\ \left.\sum_{j \in \Lambda} \sum_{k \in \Lambda} \Delta S_{j, k} \frac{\partial \boldsymbol{V}}{\partial S_{j, k}}\right|_{V=V^{*}} & \text { In uplink }\end{cases}
$$

Derivative sums in the right-hand of the last equation are given by (27) in function of the coupling matrix and vector $\Psi_{L}$. Therein, the corollary is proven.

Interpretation Lemma 3 and corollary 2 show that variation $\Delta \boldsymbol{V}$ in the power vector is mainly related to the coupling matrix and the internal load represented by $S_{j, j}$ and $D_{j}$ and vector $\boldsymbol{V}$ which could be easily computed and therefore, this estimation of $\Delta \boldsymbol{V}$ could be useful in operative networks.

Theorem 3. Suppose that the non-constrained system characterized by (21) is feasible by using vector $\boldsymbol{V}^{*}$. For small increases $\Delta S_{j, k}$ of parameters $S_{j, k}$ and $\Delta D_{j}$ of parameters $D_{j}$, we get a variation $\Delta \boldsymbol{V}$ satisfying

$$
\exists j \in \Lambda, \quad \max _{l \in \Lambda}\left(\Delta V_{l}\right) \geq \frac{1}{1-\rho(\boldsymbol{C})} \Psi_{L, j} .
$$

Proof Using corollary 2 and the same reasoning as theorem 2 and corollary 1 , we get

$$
\exists j \in \Lambda, \quad \Delta V_{j} \geq \frac{\Psi_{L, j}}{1-\rho(\boldsymbol{C})} .
$$

By using the same reasoning as corollary 1 we get (34).

Interpretation Using theorem 3, we can estimate the impact of small variations in $S_{j, k}$ and $D_{j}$ over the unconstrained transmitted power increase in downlink or the total received power in uplink. Therefore some RRM procedure could use these estimations to ameliorate system performance.

\section{APPlications of the COUPLing Matrix}

Coupling matrix approach could be easily implemented in operational systems and could be applied to different RRM and ASM methodologies since it captures interference profiles. Moreover, the properties of the coupling matrix could be used to perform the cognitive functionalities depicted in Fig. 1. Hereafter, we introduce an illustrative example of an ASM methodology using the coupling matrix in a WCDMA network with non-homogeneous spatial traffic distribution reflected by the presence of macro- and micro-cells. The objective of the proposed methodology is to detect relevant changes in traffic distribution and find a suitable allocation of WCDMA carriers to cells that satisfies QoS constraints. We highlight that the coupling matrix is for medium- and longterm use (i.e. within the scale of few seconds or more) and the elements of the matrix are averaged over a period of time. In this context, fast fluctuations (due to mobility, fast fading, etc.) are averaged and do not affect matrix accuracy. Moreover, we assume that only one carrier can be used by a base station at a given time.

\section{A. Advanced Spectrum Management Methodology}

In order to introduce the ASM methodology, we define $\boldsymbol{C}_{a}(n T)$ as the coupling matrix at each sampling time $n T$, aggregated and averaged as explained in [4]. Moreover, we derive at each sampling time $F$ partial coupling matrices $\left(\boldsymbol{C}_{a}^{(l)}(n T), \boldsymbol{C}_{a}^{(2)}(n T), \cdots, \boldsymbol{C}_{a}^{(F)}(n T)\right)$ from $\boldsymbol{C}_{\boldsymbol{a}}(n T)$. These matrices are the coupling matrices associated to the different frequencies; matrix $\boldsymbol{C}_{a}^{(f)}(n T)$ includes only the entries corresponding to cells associated to frequency carrier $f$. Moreover, we assume that $P_{\max , j}$ is the maximum available power in base station $j$.

Based on corollary 1 and its interpretation, the value of $\eta$ should be less than the maximum available power in base stations to ensure that the QoS constraints reflected by the $E_{b} / N_{0}$ are satisfied. Notice that, macro- and micro-cells have different power limitations; hence, we define new parameter $\Omega_{f}$ that considers these differences:

$$
\Omega_{f}=10 \log \max _{j \in \Lambda_{f}} \frac{\Pi_{\max , j}}{1-\rho\left(\boldsymbol{C}_{a}^{(f)}(n T)\right)}
$$

where $\Pi_{\max , j}$ is the ratio between $P_{N, j}$ and $P_{\max , j}$. As the result in Fig. 2 has showed that the maximum power is at approximately $2 \mathrm{~dB}$ higher than $\eta$, in the worst case, we define a tunable threshold margin $\vartheta_{\text {th }}$ of $2 \mathrm{~dB}$ that should not be exceeded by $\Omega_{f}$ in all frequency carriers, and the condition to provide the requested QoS levels becomes

$$
\Omega=\max _{f \in \Phi} \Omega_{f}>\vartheta_{\text {th }}
$$

The latter condition is considered as a triggering event in the developed ASM methodology whose objective is to find a proper allocation of frequency carrier to cells and is executed at both planning phase and as a reaction to traffic distribution changes. At the planning phase, the following algorithm is executed using estimated values of the coupling matrix. 
1. All matrices $\boldsymbol{C}_{a}^{(f)}$ are initiated to zero matrices: $\boldsymbol{C}_{a}^{(f)}=0$.

2. A vector $\boldsymbol{V}=\left\{V_{l}, V_{2}, \ldots, V_{k}\right\}$ is computed. The value of $V_{j}$ is computed using the sum of the corresponding rows and columns for each cell in matrix $\boldsymbol{C}$ :

$$
V_{j}=\Sigma_{l \in \Lambda-\{j\}}\left(C_{j, l}+C_{l, j}\right)
$$

3. Cells are sorted using the sum $V_{i}$ in order to take into account the impact of each cell on other cells and the latter on each cell.

4. Cell $j$ with the highest sum is associated to carrier 1 and set $\Lambda_{1}$ is updated. At this stage matrix $\boldsymbol{C}_{a}^{(1)}$ will remain null.

5. Update each sum $V_{l}$ of the remaining cells by eliminating the effect of the allocated cell $j$ :

$$
V_{l}=V_{l}-C_{j, l}-C_{l, j}
$$

6. Sort the remaining cells using the new vector $V$ and choose cell $k$ with the highest sum.

7. Estimate $\Omega_{f}$ by considering that cell $k$ is associated to all frequencies.

8. Choose frequency carrier $f$ with the lowest $\Omega_{f}$ and associate this frequency carrier to cell $k$.

9. Add cell $k$ to set $\Lambda_{f}$. and repeat from step 5

The algorithm starts by initiating $\Lambda_{f}$ to empty sets and aims at finding the spectrum allocation that minimizes $\Omega$. This is done by associating iteratively a cell to the frequency carrier that has the lowest $\Omega_{f}$. If condition (36) is detected due to traffic changes, then some cells will suffer from unacceptable QoS levels and thus the algorithm is triggered again.

\section{B. Simulation Results}

In Fig. 3, we show cell layouts and in table 1 we introduce the simulation parameters [17]. The considered scenario is formed of two frequencies, three macro-cell rings, nine dayhotspots, seven night-hotspots and 16 micro-cells at the center of the hotspots. We assume that the system considers the scenario at day-time in the planning phase and applies the ASM methodology to determine the spectrum allocation. However, at night the traffic moves from the day-hotspots to the night-hotspots. In this scenario, three approaches are considered. The first one is a static methodology where the spectrum allocation is only performed at the planning phase. The second one is a dynamic methodology where, in addition to the planning phase spectrum allocation, it performs the algorithm each time a triggering event is detected. For comparison purposes, we also present the results of the hierarchical cell structure (HCS) where one frequency carrier is associated to micro-cells and the second is associated to macro-cells. In Fig. 4, we show the evolution of the outage probability in the seven central macro-cells and the nighthotspots (i.e. the ratio of unsatisfied users to the total number of users in the considered cells). These cells experience QoS degradation in the considered scenario. Furthermore, Fig. 5 shows the evolution of the maximum total transmitted power among macro- and micro-cells. The results show that the dynamic methodology outperforms the static and the HCS methodologies by decreasing the average outage probability along the simulation by $95 \%$ and $82 \%$ respectively and the average total transmitted power in the hotspots along the simulation by $49 \%$ and $47 \%$ respectively.

\section{Practical consideration}

All needed information $\left(E_{b} / N_{0}\right.$, long-term pathlosses, spreading factors) for the computation of the coupling matrices can be obtained using the measurements collected either by base stations or mobiles. The spectral radius could be easily computed using numerical tools such as power method [16] and the complexity of the spectrum allocation algorithm is a linear function of the number of cells. Besides, the coverage of an operator could be divided into bunches to decrease algorithm complexity. Such approach was used in second generation planning tools and could be extended to third generation. In fact, each city can be considered as an independent bunch thanks to considerable distances between cities. In practice, a city can be covered in average using hundreds of macro-cells. Hence, the distribution of two or three frequencies over a city is relatively a simple task.

Moreover, if the value of $\Omega$ in the obtained spectrum allocation is higher than the old one after the convergence of the algorithm, the old spectrum allocation is conserved to prevent unnecessary changes that could affect system performance. Nevertheless, if the triggering is repeated for a long period without changing the allocation, this would mean that the network is not able to handle the existing traffic with the available resources. A solution in a heterogeneous network could be to use vertical handover or to allocate more carriers to the WCDMA system. Hence, $\Omega_{f}$ is used in the analyze functionality while $\Omega$ is used in both analyze and decide functionalities of the cognitive network. A learning functionality could be also used to adjust the value of $\vartheta_{\text {th }}$.

\section{CONCLUSIONS AND OPEN ISSUES}

In this paper, we have proposed a coupling matrix that is able to reflect both macroscopic and microscopic behavior of WCDMA system via its properties. The spectral radius of the matrix reflects the macroscopic behavior while the matrix elements reflect inter-cell interactions. We have studied coupling matrix properties and we derived interesting relations between these properties and the maximum unconstrained transmitted power as will as with its variations due to pathloss variations. Based on these relations, we have proposed a practical ASM methodology in the context of cognitive networks as an illustrative example. The ASM methodology has led to better performance than the spectrum allocation in HCS and was able to detect relevant variations in traffic distribution. Even though the proposed methodology has showed interesting results, not all coupling matrix properties have been exploited yet. For instance, the relation between system fluctuations and the spectral radius, as well as the microscopic information of the matrix, could give detailed information about each cell and could be used for different type of radio resource and spectrum management methodologies. However, this is left for future work.

\section{ACKNOWLEDGEMENT}

This work was partially performed in project E2R II which has received research funding from the Community's Sixth 
Framework program. This paper reflects only the authors' views and the Community is not liable for any use that may be made of the information contained therein. The contributions of colleagues from E2R II consortium are hereby acknowledged. This paper is also supported by the COSMOS grant (ref. TEC2004-00518, Spanish Ministry of Science and Education and European Regional Development Fund).

\section{REFERENCES}

[1] D. Maldonado, Bin Le, A. Hugine, T.W. Rondeau, and C.W. Bostian, "Cognitive radio applications to dynamic spectrum allocation: a discussion and an illustrative example," Proc of DySPAN05, 2005, p.p. 597-600.

[2] Joseph Mitola III, Cognitive Radio: An Integrated Agent Architecture for Software Defined Radio, PhD thesis, KTH, Stockholm, Sweden, 2000.

[3] R.W. Thomas, L.A. DaSilva, and A.B. MacKenzie, "Cognitive Networks," Proc. of DySPAN05, 2005, p.p.352- 360.

[4] J. Nasreddine, J. Pérez-Romero, O. Sallent, R. Agusti ,"Dynamic Spectrum Management Methodology for WCDMA Systems Based on InterCell Interaction Approach", Proc. of WPMC, 2006.

[5] J. Nasreddine, J. Pérez-Romero, O. Sallent, R. Agusti, and X. Lagrange, "A Proposal on Frequency Management Methodologies for WCDMA Systems using Cell Coupling Matrices," Proc. of VTC Fall, 2006.

[6] J. Nasreddine, J. Pérez-Romero, O. Sallent, R. Agusti, and X. Lagrange, "A Novel Frequency Management Methodology for WCDMA using Statistical Coupling Matrices," Proc of ISWCS, 2006.

[7] S. Ben Jamaa, H. Dubreil, Z. Altman, and A. Ortega, "Quality Indicator Matrices and Their Contribution to WCDMA Network Design," IEEE Transactions on Vehicular Technology, vol. 54, 2005, pp. 1114-1121.

[8] I. F. Akyildiz, W. Lee, M. C. Vuran, and S. Mohanty, "NeXt generation/dynamic spectrum access/cognitive radio wireless networks: a survey" Computer Networks Journal, (Elsevier), 2006, p.p 2127 - 2159

[9] S. A. Grandhi, R. Vijayan, D. J. Goodman, and J. Zander, "Centralized Power Control in Cellular Radio Systems," IEEE Transactions on Vehicular Technology, vol. 42, 1993, pp.66 - 468.

[10] J. Nasreddine, Radio Resource Management in TD-CDMA/TDD Systems, Ph.D. thesis, University of Rennes 1, 2005.

[11] S.V. Hanly, "Congestion measures in DS-CDMA networks" IEEE Transactions on communications, vol. 47, 1999, pp. 426 - 437.

[12] S. Grandhi, J. Zander and R. Yates, "Constrained Power Control," Int. Journal of Wireless Personal Communications, vol. 1, No. 4, 1995.

[13] M. Doob D. Cvetkovic and H. Sachs, Spectra of Graphs: Theory and Application, Academic Press, 1980.

[14] J. Pérez-Romero, O. Sallent, R. Agusti, and M. A. Diaz-Guerra, Radio Resource Management strategies in UMTS, John wiley \& Sons, 2005.

[15] R. S. Varga, Matrix Iterative Analysis. Englewood Cliffs, New Jersey, Prentice-Hall, 1962.

[16] J. W. Demmel, Applied Numerical Linear Algebra, SIAM, 1997

[17] "RF System Scenarios", 3rd Generation Partnership Project, Technical Specification Group Radio Access Network, TS 25.942, V4.2, 2002

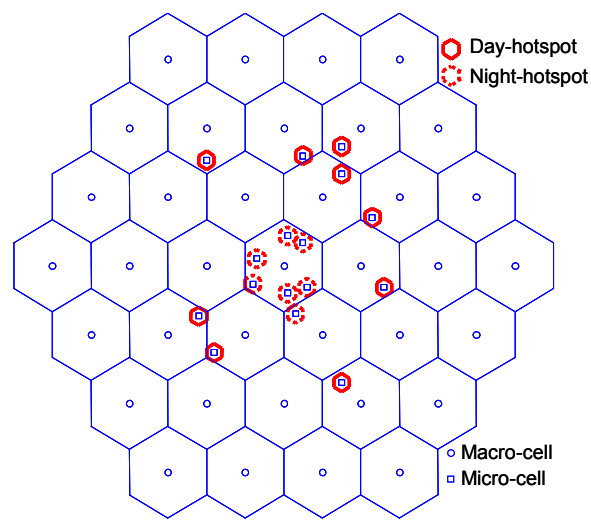

Figure 3. Layout of the macro-cells and hotspots
TABle 1 Simulation PARAmeters

\begin{tabular}{|l|l|}
\hline \multicolumn{2}{|c|}{ Macro-cell layer } \\
\hline BS pilot power & $30 \mathrm{dBm}$ \\
\hline Cell radius & $1 \mathrm{~km}$ \\
\hline Orthogonality factor & 0.4 \\
\hline BS maximum transmitted power & $43 \mathrm{dBm}$ \\
\hline \multicolumn{2}{|c|}{ Hotspot layer } \\
\hline Micro-cell BS pilot power & $20 \mathrm{dBm}$ \\
\hline Cell radius & $0.2 \mathrm{Km}$ \\
\hline Orthogonality factor & 0.06 \\
\hline BS maximum transmitted power & $33 \mathrm{dBm}$ \\
\hline \multicolumn{2}{|c|}{ Common parameters } \\
\hline Pathloss model & $128.1+37.6 \times \log _{10} d(\mathrm{Km})$ \\
\hline Background noise & $-98 \mathrm{dBm}$ \\
\hline UE maximum power for macrocells & $30 \mathrm{dBm}(20$ for micro $)$ \\
\hline Transmitted power range & $25 \mathrm{~dB}$ \\
\hline$E_{\mathrm{b}} / N_{0}$ target & $6 \mathrm{~dB}$ \\
\hline Spreading factor & $23 \mathrm{~dB}$ \\
\hline Shadowing factor deviation & $7 \mathrm{~dB}$ \\
\hline Shadowing factor cross-correlation & 0.5 \\
\hline Sampling period $T$ & $100 \mathrm{~s}$ \\
\hline Macro-cell density & $27 \mathrm{mobiles} / \mathrm{Km}^{2}$ \\
\hline Micro-cell density & $810 \mathrm{mobiles} / \mathrm{Km}^{2}$ \\
\hline Power control & Perfect power control \\
\hline
\end{tabular}

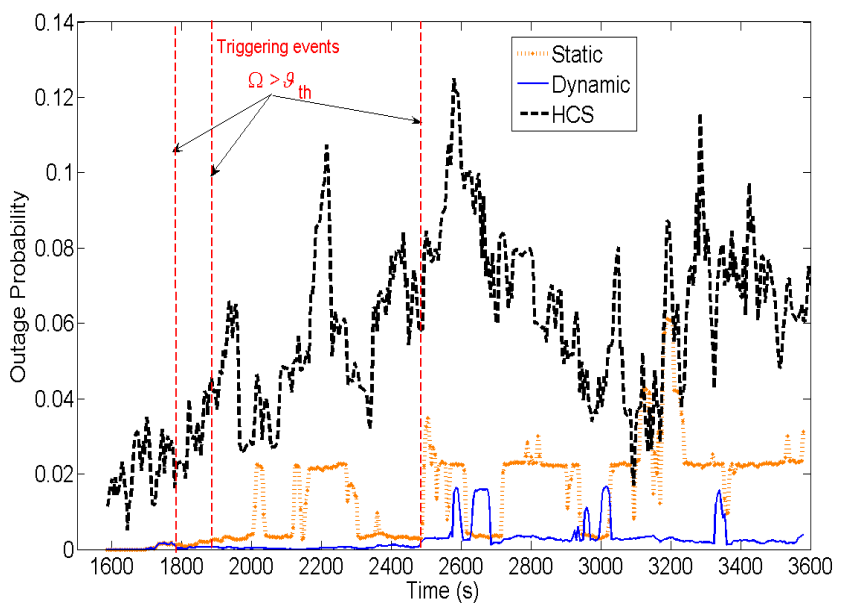

Figure 4. Outage probability of the 7 central cells and night hotspots

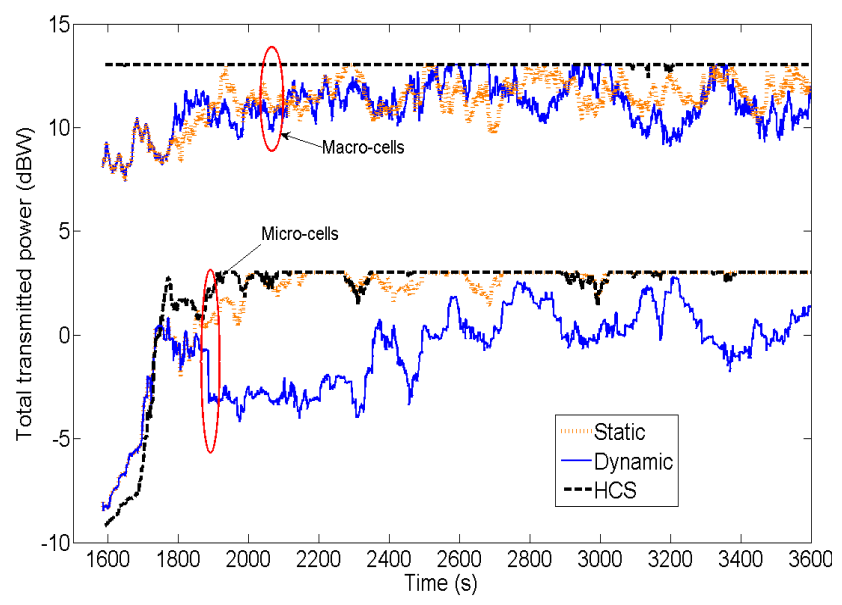

Figure 5. Maximum total transmitted power of the 7 central cells and night hotspots 